\title{
Mitteilungen der Berliner Gesellschaft
}

fuir Psychiatrie und Neurologie e. V.

\section{Ausschreibung: Promotionspreis der BGPN}

Auch in diesem Jahr verleiht die Berliner Gesellschaft für Psychiatrie und Neurologie den Promotionspreis an die 2 besten eingereichten Promotionen des letzten Jahres aus den Gebieten der Psychiatrie und Neurologie. Die Preisträger erhalten ein Preisgeld von je 1000 Euro und die Möglichkeit, ihre Arbeiten bei der wissenschaftlichen Veranstal- tung der BGPN am 13.05.2020 vorzustellen. Bewerber müssen ihre Promotion bis zum 31.12.2019 an einer Berliner Institution abgeschlossen haben. Die Abgabefrist endet am 15.03.2020. Bitte senden Sie Ihre Arbeiten an den Schriftführer der Gesellschaft: stephan.koehler@charite.de

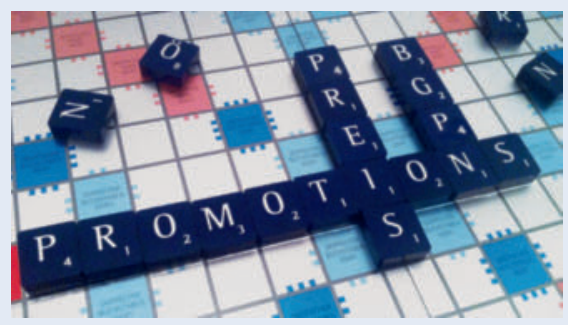

GRUSS ZUM NEUEN JAHR

Die Berliner Gesellschaft für Psychiatrie und Neurologie e. V. wünscht Ihnen ein gesundes neues Jahr 2020!

\section{Ort: Kaiserin-Friedrich-Haus, Robert-Koch-Platz 7, 10115 Berlin}

Iktale Phänomene in Psychiatrie und Neurologie

- Diagnostik und Therapie dissoziativer Anfälle;

Prof. Straub und Dr. Fonseca, Bernau

- Transiente globale Amnesie; Prof. Dr. Bartsch, Kiel

- Tourette-Syndrom/Tics; Dr. Ganos, Berlin

- Synkopen versus Anfälle; Prof. Dr. Haverkamp, Charité

- Synaesthesien; Dr. Markus Zedler, Hannover

Teilnahme kostenfrei.

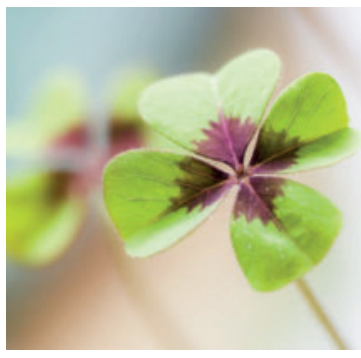

13.05.2019

- Verleihung des Promotionspreises der BGPN und Vorträge der Promotionspreisträger

- NMO-Spektrum-Erkrankungen; Prof. Dr. Lutz, Charité

10.06.2019

- Augenbewegungsstörungen als Symptom und diagnostischer Marker; PD Dr. Ostendorf, Berlin;

- Griesinger-Vortrag: Psychiatrie im Nationalsozialismus: Erinnerung und Verantwortung; Prof. Schneider, Düsseldorf
IMPRESSUM

Prof. Dr. Tom Bschor Redaktion: Dr. Anja M. Bauer Berliner Gesellschaft für Psychiatrie und Neurologie e. V.

Schlosspark-Klinik, Abteilung für Psychiatrie

Heubnerweg 2, 14059 Berlin info@bgpn.de,www.bgpn.de 